\title{
SISTEMA LIMPO EM LINHA PARA EXTRAÇÃO EM FASE SÓLIDA DE CONTAMINANTES EMERGENTES EM ÁGUAS NATURAIS
}

\author{
Fernando F. Sodré*, Marco Antonio F. Locatelli e Wilson F. Jardim \\ Instituto de Química, Universidade Estadual de Campinas, CP 6154, 13084-862 Campinas - SP, Brasil
}

Recebido em 13/3/09; aceito em 19/6/09; publicado na web em 27/11/09

\begin{abstract}
AN IN-LINE CLEAN SYSTEM FOR THE SOLID-PHASE EXTRACTION OF EMERGING CONTAMINANTS IN NATURAL WATERS. A solid-phase in-line extraction system for water samples containing low levels of emerging contaminants is described. The system was specially developed for large volume samples (up to 4 L) using commercial solid-phase extraction (SPE) cartridges. Four sets containing PTFE-made connectors, brass adapters and ball valves were used to fit SPE cartridges and sample bottles to a 4-port manifold attached to a $20 \mathrm{~L}$ carboy. A lab-made vacuum device was connected to the manifold cap. The apparatus is robust and less expensive than the typical available system. Its also provides less experimental handling, avoiding cross contamination and sample losses.
\end{abstract}

Keywords: solid-phase extraction; emerging contaminants; large volume samples.

\section{INTRODUÇÃO}

Dentro de uma sequência analítica, o tratamento da amostra constitui etapa importante na qual os analitos são levados a uma forma passível de quantificação de acordo com o método analítico escolhido. ${ }^{1}$ Além disso, esta etapa pode ser a mais demorada e dispendiosa de uma sequência analítica. Um método analítico ideal deve possuir mínima manipulação experimental, oferecer máxima segurança operacional e não permitir contaminação ou perda dos constituintes de interesse. ${ }^{2}$ Todos estes aspectos estão diretamente associados à etapa de tratamento da amostra.

Métodos voltados à determinação de contaminantes emergentes em águas naturais estão, em sua maioria, condicionados a uma etapa de extração e pré-concentração. ${ }^{3}$ Inúmeras estratégias têm sido adotadas para este fim, sendo que grande parte dos trabalhos faz uso de métodos de extração em fase sólida (SPE, solid phase extraction) com cartuchos disponíveis comercialmente. ${ }^{3,4} \mathrm{O}$ uso de cartuchos para a extração de contaminantes emergentes tem levado à obtenção de excelentes índices de recuperação em diferentes faixas de concentração. ${ }^{4,5}$

Produtos farmacêuticos e de higiene pessoal, hormônios, derivados do colesterol e alguns sub-produtos industriais têm sido considerados contaminantes emergentes. ${ }^{6}$ Em águas naturais, estes contaminantes surgem, principalmente, a partir do descarte de efluentes industriais e/ou domésticos ${ }^{7} \mathrm{e}$, mesmo sob concentrações baixas (ng L ${ }^{-1}$ a pg L L ${ }^{-1}$ ), podem provocar efeitos nocivos em organismos vivos. Pouquíssimos estudos têm abordado a ocorrência de contaminantes emergentes em águas brasileiras.

Em águas naturais da região de Campinas, por exemplo, contaminantes emergentes foram determinados após extração em fase sólida empregando-se cartuchos SPE comercialmente disponíveis. ${ }^{8}$ Embora a etapa de pré-concentração dos compostos de interesse tenha sido feita seguindo-se recomendações preconizadas na literatura especializada, algumas dificuldades operacionais foram enfrentadas durante o desenvolvimento dos trabalhos supramencionados, o que motivou a busca por melhorias desta etapa da sequência analítica. O sistema operacional mais comumente utilizado durante a etapa de pré-concentração emprega uma série de equipamentos e é detalhado na Figura 1.

No sistema mostrado na Figura 1, a extração é realizada com o auxílio de três equipamentos principais: uma bomba de vácuo, uma bomba peristáltica e um manifold a vácuo de 12 portas. A amostra, contida em uma garrafa de vidro âmbar, é continuamente gotejada

*e-mail: ffsodre@iqm.unicamp.br para o cartucho SPE com o auxílio da bomba peristáltica. É importante mencionar que para a extração de $n$ amostras são necessários $n$ canais disponíveis na bomba peristáltica. Tubos flexíveis de bombeamento, confeccionados com materiais diversos, são empregados para conduzir a amostra até o cartucho SPE. No manifold a vácuo, o cartucho de extração é afixado e a amostra é aspirada para recipientes acondicionados no interior do manifold. O vácuo no interior deste equipamento é estabelecido com o auxílio da bomba de vácuo.

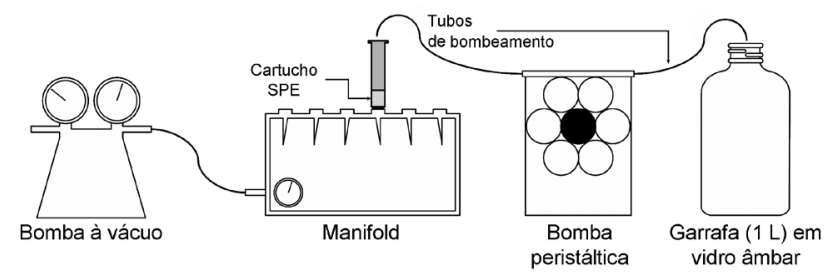

Figura 1. Esquema mostrando o procedimento utilizado para extração em fase sólida de contaminantes emergentes em águas naturais

Embora tenha se mostrado eficaz para o processamento de amostras de águas naturais, ${ }^{8}$ procedimentos baseados no uso do sistema mostrado da Figura 1 demandam muitos cuidados e, consequentemente, a presença do analista durante toda a etapa de extração. Alguns dos cuidados necessários, assim como as principais desvantagens associadas a estes procedimentos são: o controle combinado da vazão da amostra (bomba peristáltica) e da intensidade do vácuo, uma vez o ajuste de ambos os parâmetros deve ser realizado em sincronia; a contaminação cruzada por meio da lixiviação de compostos orgânicos contidos nos tubos flexíveis de bombeamento; a possível contaminação da amostra uma vez que o cartucho de extração fica em contato com o ar; a necessidade de interromper a extração de tempos em tempos para retirar os béqueres do interior do manifold (para a extração de $1 \mathrm{~L}$ de amostra o sistema é interrompido três vezes); a utilização prolongada da bomba de vácuo, o que pode provocar a falha momentânea ou permanente do equipamento; e o elevado custo associado ao material empregado. Estima-se que o preço dos três equipamentos supramencionados seja de mais de $\mathrm{R} \$ 15.000,00$.

Levando-se em conta as desvantagens apresentadas pelo sistema da Figura 1, um novo sistema de extração é proposto. Considerando-se que a presença de contaminantes emergentes pode ser relevante mesmo 
sob concentrações extremamente baixas, o objetivo deste trabalho foi desenvolver um sistema operacional alternativo capaz de processar volumes elevados de amostra de maneira rápida, contínua e, principalmente, limpa. As mudanças propostas neste trabalho visam apenas facilitar a operacionalidade da etapa de extração sem alterar parâmetros de mérito inerentes ao método analítico empregado.

\section{CONFECÇÃO DE PEÇAS EM PTFE}

Como forma de evitar a contaminação da amostra e, ao mesmo tempo, permitir a passagem contínua e ininterrupta da amostra pelo cartucho de extração, optou-se pela construção de um sistema limpo em linha similar ao adotado pela U. S. Geological Survey (USGS). ${ }^{9}$ Ao contrário do método USGS, o sistema proposto neste projeto lança mão de materiais voltados a reduzir ao máximo a contaminação da amostra por meio da lixiviação de compostos orgânicos contaminantes. Assim, o sistema foi construído apenas com tarugos de Teflon ${ }^{\circledR}$ (PTFE - polytetrafluoroethylene) e conexões de latão e aço inox. A primeira etapa da construção do sistema de extração envolveu o acondicionamento do cartucho SPE em peças de PTFE. A Figura 2 mostra peças de PTFE confeccionadas para afixar as partes superior e inferior de cartuchos de extração comercialmente disponíveis.

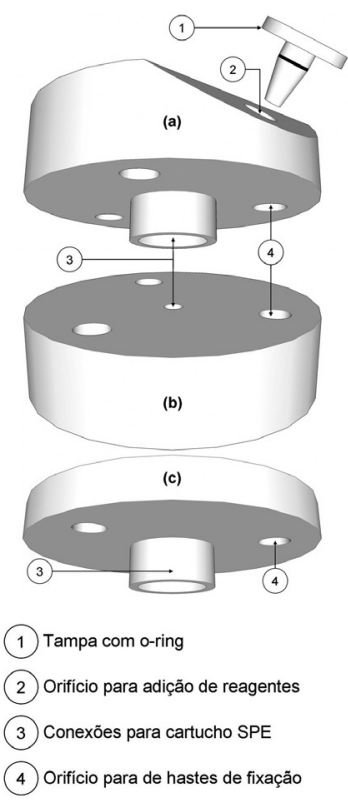

Figura 2. Peças confeccionadas em PTFE para afixação de cartuchos de extração em fase sólida; (a) peça para encaixe superior, $(b)$ peça para encaixe inferior e (c) peça para encaixe in tandem

A peça $a$ mostrada na Figura 2 consiste de um bloco cilíndrico $(\varnothing$ $=50 \mathrm{~mm}$ ) contendo, em uma das faces, uma conexão (3) para encaixe da parte superior de um cartucho SPE comum $(6 \mathrm{~mL})$. Na face oposta existe um orifício rosqueado (não mostrado) para o encaixe de roscas $N P T$ de $1 / 8$ de polegada. Na parte superior da peça $a$ existe também um orifício (2) destinado à adição de reagentes para o interior no cartucho de extração. Este orifício, que contém uma tampa móvel (1) é especialmente importante para permitir a ativação do material presente nos cartuchos. O bloco ainda possui três furos (4) voltados à passagem de hastes de fixação.

A extremidade inferior do cartucho SPE também é afixado em uma das faces da peça $b$, a qual possui dimensões similares à peça $a \mathrm{e}$ também contém, em uma das faces, um orifício para o encaixe de roscas NPT de $1 / 8$ de polegada. As peças de PTFE foram confeccionadas para comportar cartuchos com diâmetro interno de $13 \mathrm{~mm}$, mais usados corriqueiramente. Entretanto, outros cartuchos podem ser empregados variando-se a dimensão das conexões (3) mostradas na Figura 2.

O sistema de extração também foi projetado para a realização de extração em linha in tandem, onde dois cartuchos são ligados consecutivamente. Este tipo de extração é muito empregado para a inclusão de uma etapa adicional de clean up de amostras complexas contendo teores elevados de matéria orgânica dissolvida. ${ }^{10}$ Para possibilitar a construção do sistema in tandem a peça $c$ foi confeccionada contendo, em ambas as faces, conexões para o encaixe de um cartucho SPE comum. A Figura 3 mostra dois conjuntos completos de extração formados a partir da conexão entre as peças de PTFE e cartuchos de extração simples e in tandem.

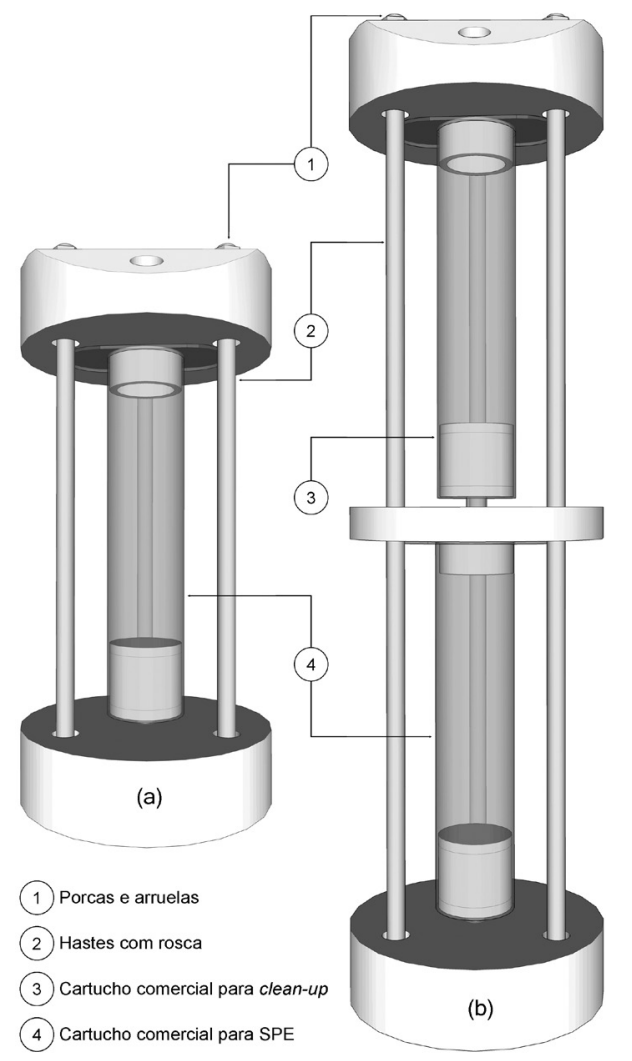

Figura 3. Conjunto para extração em fase sólida (a) e para extração em fase sólida in tandem (b) empregando-se cartuchos disponíveis comercialmente

Na Figura 3 é possível visualizar os conjuntos para a extração em fase sólida construídos com um e dois cartuchos. Ambos os conjuntos são montados com o auxílio de hastes rosqueadas, porcas e arruelas, como forma de evitar vazamentos e contaminações externas.

\section{CONSTRUÇÃO DE SISTEMA DE EXTRAÇÃO EM LINHA}

Um esquema representativo do sistema de extração proposto neste trabalho é mostrado na Figura 4.

Procedimentos fundamentados no uso do sistema proposto baseiamse na passagem da amostra por um cartucho SPE conectado em linha, em sistema fechado e sob vácuo. O vácuo é criado com o auxílio de uma bomba centrífuga de $1 / 4 \mathrm{CV}$ que bombeia água para um injetor Venturi comercial. A sucção gerada pelo efeito Venturi pode ser similar à obtida por uma bomba elétrica de vácuo. A água empregada para a criação do vácuo é acondicionada em uma caixa plástica de $18 \mathrm{~L}$ de capacidade.

A parte central do injetor Venturi é ligado a uma bombona de plástico reforçado para vácuo (Nalgene) com capacidade para armazenar até 20 L de água. A tampa da bombona foi confeccionada em nylon e possui orifícios para conectar até quatro sistemas individuais de extração. 


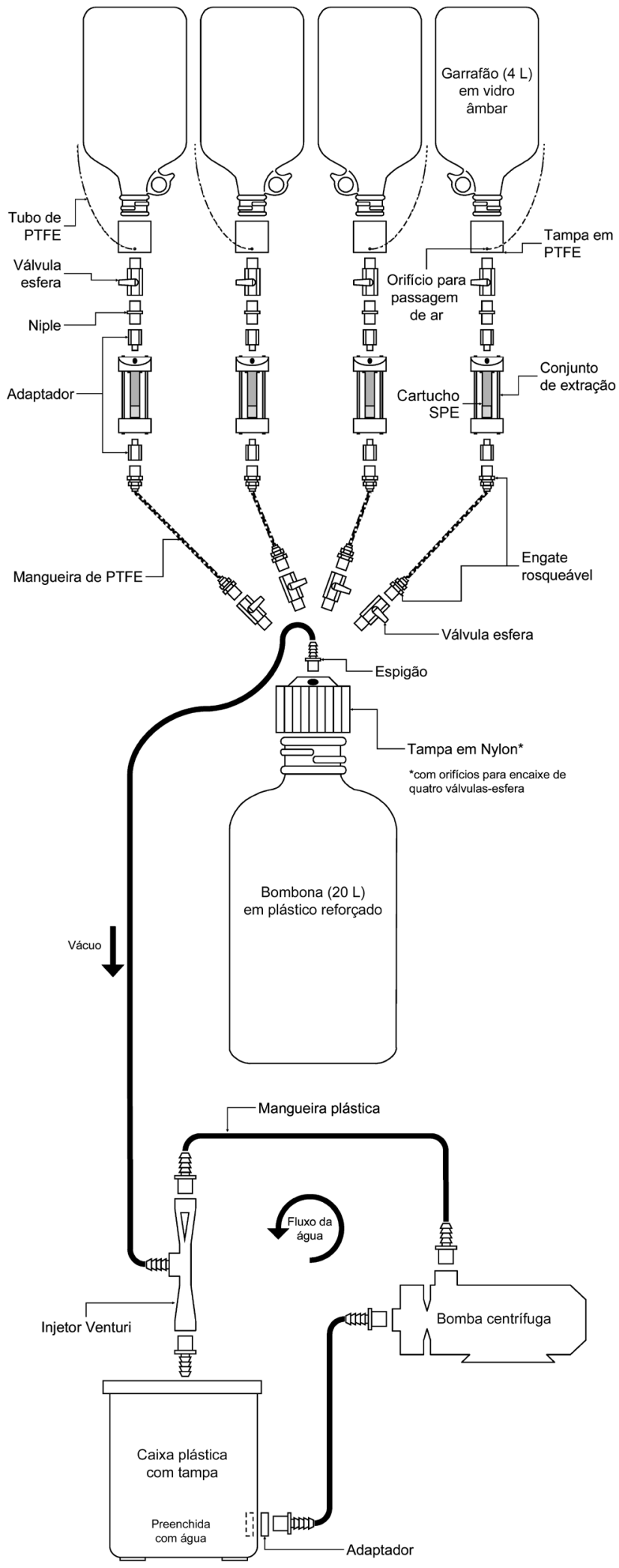

Figura 4. Sistema completo empregado para extração em fase sólida de contaminantes emergentes em amostras de águas naturais

Cada sistema individual de extração é formado por uma série de peças e conexões que interligam, em linha, o garrafão de vidro contendo a amostra à bombona mantida sob vácuo, passando pelo conjunto de extração. Na Figura 4 pode-se observar que cada sistema foi original- mente construído para a utilização de garrafões de $4 \mathrm{~L}$ de capacidade. Em cada garrafão existe uma tampa de PTFE contendo orifício para conectar válvulas-esfera simples com rosca NPT de $1 / 4$ de polegada. A válvula permite o controle da passagem da amostra até o cartucho SPE e é especialmente importante para evitar a passagem da amostra durante a etapa de ativação do material adsorvente contido no interior do cartucho.

Um conector (niple) reto de latão é utilizado para interligar a válvula-esfera ao adaptador M/F (1/4 x 1/4). Este, por sua vez é conectado ao conjunto de extração. Na base do conjunto de extração um segundo adaptador $\mathrm{M} / \mathrm{F}$ é conectado a um engate pneumático com rosca de 1/4 x 1/4 de polegada. Neste engate tem-se a ligação de uma mangueira de plástico rígido (PTFE ou material similar) utilizada para transportar a amostra processada até a bombona mantida sob vácuo. Na tampa de nylon da bombona, cada sistema individual de extração é conectado por meio de uma válvula-esfera interligada a um novo engate pneumático. Desta maneira, é possível controlar, individualmente, o vácuo de cada um dos quatro sistemas de extração. Além disso, esta configuração permite que diferentes tipos de extração sejam realizados em cada canal e em momentos diferentes. Fotos dos sistemas de extração são mostrados nas Figuras $1 \mathrm{~S}$ e $2 \mathrm{~S}$, material suplementar.

Inúmeros testes foram realizados para verificar a ocorrência de vazamentos e para conhecer a faixa de vazão do sistema. Os primeiros testes evidenciaram a necessidade de promover a entrada de ar na parte superior do sistema como forma de manter o fluxo da amostra contínuo e ininterrupto. Desta forma, optou-se pela introdução de um orifício na tampa de cada garrafão de $4 \mathrm{~L}$ para afixação de pequenos tubos de PTFE. A outra extremidade do tubo permanece em contato com o ar ou é direcionada para microambientes saturados com $\mathrm{N}_{2}$ à pressão atmosférica. A utilização dos tubos de PTFE permitiu um controle total da vazão do sistema que pôde ser mantida constante em uma faixa de 6 a $30 \mathrm{~mL} \mathrm{~min}{ }^{-1}$. Além disso, observou-se que o nível hidrostático da amostra que passa pelo cartucho permanece inalterado durante todo o procedimento de extração. Este aspecto é particularmente importante, pois permite que a extração seja realizada de maneira ininterrupta, sem interveniência do analista.

Em trabalho recente, ${ }^{11}$ o conjunto in tandem foi empregado, com sucesso, para otimizar a extração de oito antibióticos em amostras de águas superficiais da Bacia do Rio Atibaia. Os resultados mostraram a presença de cefalexina (2422 ng L-1), amoxicilina (1284 $\left.\mathrm{ng} \mathrm{L}^{-1}\right)$, ciprofloxacin (133 $\left.\mathrm{ng} \mathrm{L}^{-1}\right)$ e norfloxacin $\left(51 \mathrm{ng} \mathrm{L}^{-1}\right)$ em amostras coletadas no Ribeirão Anhumas, evidenciando o elevado impacto associado ao descarte de esgoto bruto a partir de regiões densamente urbanizadas. ${ }^{11}$

\section{AVALIAÇÃO DE CUSTOS}

Além de atender a todas as expectativas quanto ao desenvolvimento de um sistema alternativo para a extração, o aparato utilizado mostrou-se menos oneroso que aquele mostrado na Figura 1. A Tabela 1 mostra o preço de cada um dos itens empregados para a construção do sistema de extração mostrado na Figura 4, com exceção dos garrafões de $4 \mathrm{~L}$ (frascos de solvente reaproveitados).

Pode-se observar que o sistema de extração desenvolvido neste trabalho é, aproximadamente, 12 vezes mais barato do que aquele baseado no uso de bomba de vácuo, manifold e bomba peristáltica (Figura 1). Além disso, este sistema pode ser modificado para o processamento de oito amostras com $4 \mathrm{~L}$ cada. Neste caso, o vácuo criado no injetor Venturi pode ser dividido para duas bombonas. Com esta configuração o preço total do sistema é de cerca de R \$ 1900,00. Embora pareça mais cara, esta ampliação do sistema de extração promove a redução do valor da extração por litro de amostra (de $\mathrm{R} \$ 73,46$ para $\mathrm{R} \$ 59,40$ ), uma vez que todo o aparato utilizado para criação de vácuo é compartilhado entre duas bombonas de $20 \mathrm{~L}$. 
Tabela 1. Materiais utilizados para a confecção de sistema de extração, em linha, de contaminantes emergentes EM águas naturais

\begin{tabular}{|c|c|c|c|}
\hline Item & Descrição & Quantidade & Preço $(\mathrm{R} \$)$ \\
\hline 1 & $\begin{array}{l}\text { Bombona Nalgene } \\
\text { (Cat. № 2226-0050) }\end{array}$ & 1 un & 340,00 \\
\hline 2 & $\begin{array}{l}\text { Bomba centrífuga } \\
1 / 2 \mathrm{CV} \text { (Dancor) }\end{array}$ & 1 un & 275,00 \\
\hline 3 & Peças em PTFE* & 12 un & 135,00 \\
\hline 4 & $\begin{array}{l}\text { Injetor Venturi com } \\
\text { válvula anti-refluxo }\end{array}$ & 1 un & 85,00 \\
\hline 5 & $\begin{array}{l}\text { Válvula esfera mini } \\
\text { M/F } 1 / 4 B S P\end{array}$ & 8 un & 84,56 \\
\hline 6 & Tampa em nylon* & 1 un & 80,00 \\
\hline 7 & $\begin{array}{l}\text { Engate pneumático rosqueável } \\
\qquad 1 / 4 \times 1 / 4 N P T\end{array}$ & 8 un & 43,84 \\
\hline 8 & Adaptador M/F $1 / 8$ x $1 / 4$ & 8 un & 33,92 \\
\hline 9 & Caixa plástica com tampa & 1 un & 29,90 \\
\hline 10 & Espigão & 6 un & 15,24 \\
\hline 11 & Mangueira plástica & $2 \mathrm{~m}$ & 16,00 \\
\hline 12 & $\begin{array}{l}\text { Conector (niple) } \\
\text { reto } \mathrm{M} / \mathrm{M} 1 / 4 N P T\end{array}$ & 4 un & 14,00 \\
\hline 13 & Mangueira PTFE & $1 \mathrm{~m}$ & 7,50 \\
\hline 14 & Tubo de PTFE & $1 \mathrm{~m}$ & 4,50 \\
\hline 15 & Porcas $(U N C 5 / 32)$ e arruelas & 24 un & 3,27 \\
\hline 16 & Adaptador para espigão & 2 un & 3,15 \\
\hline 17 & Hastes roscadas $U N C 5 / 32$ & $1 \mathrm{~m}$ & 2,56 \\
\hline \multirow[t]{2}{*}{18} & Fitas PTFE $50 \mathrm{~m}$ & 1 un & 1,96 \\
\hline & & TOTAL & 1175,40 \\
\hline
\end{tabular}

* inclui preço da peça bruta e serviço de torneamento.

\section{CONCLUSÕES}

O sistema de extração em linha desenvolvido neste trabalho mostrou-se adequado para o processamento de volumes elevados de amostra. Permitiu a utilização de um ou mais cartuchos de extração disponíveis comercialmente sem perdas de amostra. Além disso, mostrou-se mais rápido e menos oneroso que o sistema outrora empregado. Finalmente, o novo sistema de extração também contribuiu para a redução da manipulação experimental e, consequentemente, dos riscos de contaminação cruzada.

\section{MATERIAL SUPLEMENTAR}

No material suplementar, disponível gratuitamente em http:// quimicanova.sbq.org.br na forma de arquivo PDF, são mostradas fotos do sistema de extração para um e dois cartuchos SPE em linha (Figura 1S), assim como detalhes da tampa da bombona 20 L (Figura 2S), utilizada como manifold de 4 portas.

\section{AGRADECIMENTOS}

À Fundação de Amparo à Pesquisa do Estado de São Paulo por meio dos processos 2007/59208-9 (PD País) e 2007/58449-2 (Projeto Temático).

\section{REFERÊNCIAS}

1. Luque de Castro, M. D.; da Silva, M. P.; Trends Anal. Chem. 1997, 16, 16; Dean, J. R.; Xiong, G.; Trends Anal. Chem. 2000, 19, 553; Priego-Capote, F.; Luque de Castro, M. D.; Trends Anal. Chem. 2004, 23, 644; Zougagh, M.; Valcárcel, M.; Rios, A.; Trends Anal. Chem. 2004, 23, 399; Ramos, L.; Ramos, J. J.; Brinkman, U. A. Th.; Anal. Bioanal. Chem. 2005, 381, 119; Ellis, S. G.; Booij, K.; Kaputa, M.; Chemosphere 2008, 72, 1112.

2. Krug, F. J. Em Métodos de Decomposição de Amostras; Krug, F. J., ed.; Workshop sobre Preparo de Amostras: São Paulo, 2004, cap. 1.

3. Ternes, T. A.; Trends Anal. Chem. 2001, 20, 419; Miao, X.; Bishay, F.; Chen, M.; Metcalfe, C. D.; Environ. Sci. Technol. 2004, 38, 3533; Hao, C.; Lissemore, L.; Nguyen, B.; Kleywegt, S.; Yang, P.; Solomon, K.; Anal. Bioanal. Chem. 2006, 384, 505; Filali-Meknassi, Y.; Auriol, M.; Adams, C. D.; Surampalli, R. Y.; Water Environ. Res. 2007, 79, 687; Fontanals, N.; Marcé, R. M.; Borrull, F.; J. Chromatogr., A 2007, 1152, 14; Richardson, S. D.; Anal. Chem. 2008, 80, 4373; Zhao, J. L.; Ying, G. G.; Wang, L., Yang, J. F.; Yang, X. B.; Yang, L. H.; Li, X.; Sci. Total Environ. 2009, 407, 962; Kuster, M.; de Alda, M. L.; Barceló, D.; J. Chromatogr., A 2009, 1216, 520 .

4. Castillo, M.; Alonso, M. C.; Riu, J.; Barceló, D.; Environ. Sci. Technol. 1999, 33, 1300; Van De Steene, J. C.; Lambert, W. E.; J. Chromatogr., A 2008, 1182, 153; Kasprzyk-Hordern, B.; Dinsdale, R. M.; Guwy, A. J.; J. Chromatogr., A 2007, 1161, 132.

5. Arditsoglou, A.; Voutsa, D.; Environ. Sci. Pollut. Res. 2008, 15, 228 ; Conley, J. M.; Symes, S. J.; Kindelberger, S. A.; Richards, S. M.; J. Chromatogr., A 2008, 1185, 206.

6. Kolpin, D. W.; Furlong, E. T.; Meyer, M. T.; Thurman, E. M.; Zaugg, S. D.; Barber, L. B.; Buxton, H. T.; Environ. Sci. Technol. 2002, 36, 1202; Schwarzenbach, R. P.; Escher, B. I.; Fenner, K.; Hofstetter, T. B.; Johnson, C. A.; von Gunten, U.; Wehrli, B.; Science 2006, 313, 1072; Glassmeyer, S. T.; Furlong, E. T.; Kolpin, D. W.; Cahill, J. D.; Zaugg, S. D.; Werner, S. L.; Meyer, M. T.; Kryak D. D.; Environ. Sci. Technol. 2005, 39, 5157; Focazio, M. J.; Kolpin, D. W.; Barnes, K. K.; Furlong, E. T.; Meyer, M. T.; Zaugg, S. D.; Barber, L. B.; Thurman, M. E.; Sci. Total Environ. 2008, 402, 201.

7. Heberer, T.; J. Hydrol. 2002, 266, 175; Heberer, T.; Reddersen, K.; Mechlinski, A.; Water Sci. Technol. 2002, 46, 81; Bendz, D.; Paxéus, N. A.; Ginn, T. R.; Loge, F. J.; J. Hazard. Mater. 2005, 122, 195; Khanal, S. K.; Xie, B.; Thompson, M. L.; Sung, S.; Ong, S. K.; van Leeuwen, J. H.; Environ. Sci. Technol. 2006, 40, 6537; Choi, K.; Kim, Y.; Park, J.; Park, C. K.; Kim, M. Y.; Kim, H. S.; Kim, P.; Sci. Total Environ. 2008, 405, 120.

8. Sodré, F. F.; Montagner, C. C.; Locatelli, M. A. F.; Jardim, W. F.; J. Braz. Soc. Ecotoxicol. 2007, 2, 187; Sodré, F. F.; Locatelli, M. A. F.; Jardim, W. F.; Water, Air, Soil Pollut., doi 10.1007/s11270-009-0086-9.

9. Zaugg, S. D.; Smith, S. G.; Schroeder, M. P.; Barber, L. B.; Burkhardt, M. R.; Methods of Analysis by the U. S. Geological Survey National Water Quality Laboratory - Determination od Wastewater Compounds by Polystyrene-Divinylbenzene Solid-Phase Extraction and CapillaryColumn Gas Chromatography/Mass Spectrometry, U. S. Geological Survey Water-Resources Investigations: Denver, 2002, Report 01-4186.

10. Castillo, M.; Ventura, F.; Barceló, D.; Waste Manage. 1999, 19, 101; Huang, S. B.; Stanton, J. S.; Lin, Y.; Yokley, R. A.; J. Agric. Food Chem. 2003, 51, 7252; Kay, P.; Blackwell, P. A.; Boxall, A. B. A.; Chemosphere 2005, 60, 427; Blackwell, P. A.; Kay, P.; Boxall, A. B. A.; Chemosphere 2007, 67, 292.

11. Locatelli, M. A. F.; Sodré, F. F.; Jardim, W. F.; Arch. Environ. Contam. Toxicol., submetido. 


\section{SISTEMA LIMPO EM LINHA PARA EXTRAÇÃO EM FASE SÓLIDA DE CONTAMINANTES EMERGENTES EM} ÁGUAS NATURAIS

Fernando F. Sodré*, Marco Antonio F. Locatelli e Wilson F. Jardim

Instituto de Química, Universidade Estadual de Campinas, CP 6154, 13084-862 Campinas - SP, Brasil

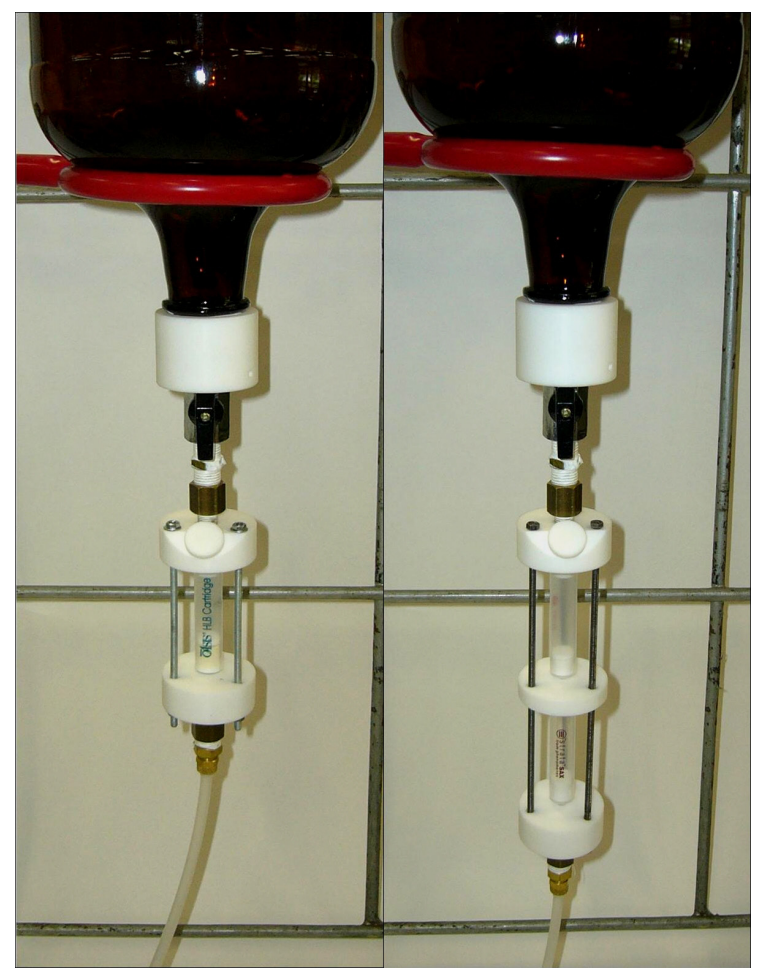

Figura 1S. Conjuntos de extração para um e dois cartuchos (in tandem) em linha

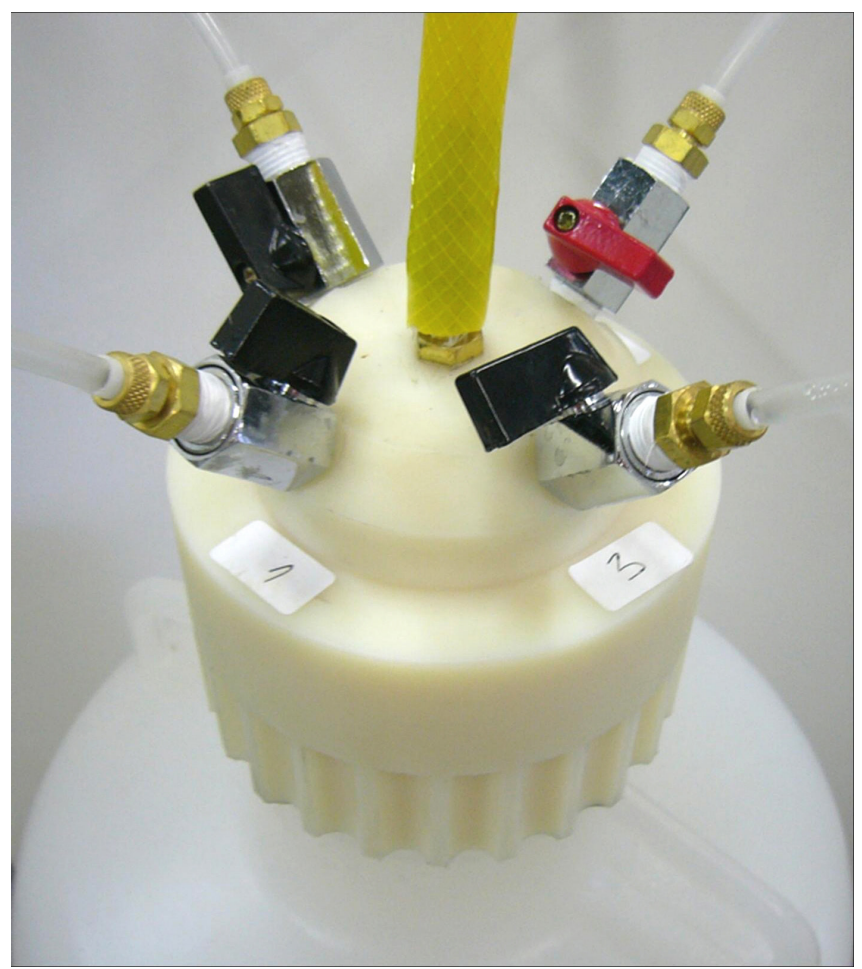

Figura 2S. Tampa de nylon (manifold) utilizado na bombona de $20 \mathrm{~L}$ 